\title{
DIÁlOGOS DE SABERES NA PROMOÇÃO DA SAÚDE E PREVENÇÃO DE AGRAVOS RELACIONADOS À COVID-19
}

\author{
Najla de Oliveira Cardozo ${ }^{1}$ \\ Barbara Cassetari Sugizaki ${ }^{1}$
}

\begin{abstract}
Resumo
No contexto da pandemia da COVID-19 este trabalho procurou refletir sobre promoção e prevenção à saúde a partir de diferentes saberes. $O$ trabalho foi delineado por meio de uma revisão bibliográfica baseada em evidências científicas e saberes tradicionais, na perspectiva multissistêmica da saúde. Englobou os aspectos nutricionais, atividade física e a integralidade do cuidado em saúde em uma abordagem holística, transcendendo o conhecimento cientificamente produzido para a discussão dos conhecimentos tradicionais e outros elementos culturais de extrema importância. Diante do acúmulo de saberes em saúde e nutrição enfatizase a reflexão do arsenal biocultural na compreensão da saúde integral frente aos desafios advindos da pandemia.
\end{abstract}

Palavras-chave: pandemia; promoção da saúde; nutrição; cultura alimentar; integração de saberes.

\section{Introdução}

Em um contexto no qual a pandemia da COVID-19 desencadeia mudanças sociais, para além das práticas sanitárias e modificações de hábitos no campo da saúde, este trabalho propõe uma reflexão sobre práticas promotoras de saúde e bem-estar, as quais possam contribuir positivamente neste cenário. No texto buscaremos fazer uma análise sobre as tendências atuais da pesquisa científica na área da nutrição e como o conhecimento existente, mesmo que de forma genérica, pode beneficiar a população submetida à ameaça da COVID-19. Na sequência apresentaremos outros tipos de conhecimento que tradicionalmente são empregados para melhorar a saúde e o bem-estar das pessoas. Dessa forma, pretende-se promover o diálogo de saberes já validados pela ciência e saberes tradicionais entre as práticas promotoras de saúde e a prevenção de doenças. Nesse sentido, este trabalho parte da pergunta: como pensar na promoção da saúde em um contexto da pandemia da COVID-19?

\footnotetext{
${ }^{1}$ Programa de pós-graduação em Alimentos e Nutrição da Universidade Estadual Paulista Júlio de Mesquita Filho (UNESP). Faculdade de Ciências Farmacêuticas. ORCID: 0000-0001-5000-6935/ 0000-0001-5152-6246

E-mail: najla_oc@hotmail.com
} 


\section{Metodologia}

Este trabalho teve o objetivo de discutir a importância da cultura alimentar, da alimentação e da atividade física como grandes aliados na rotina da população para a promoção da saúde e prevenção da COVID-19. O trabalho consistiu-se de uma revisão bibliográfica com interpretação dos dados pertinentes. Para isso, houveram dois grupos de referencial bibliográfico: (1) artigos relacionados à evidências científicas em doenças sistêmicas, nutrição e atividade física nas bases de dados Medline, Lilacs, Google Acadêmico, Spell e Scielo por meio de artigos originais e revisões sistemáticas, publicados nas últimas duas décadas em inglês, espanhol e português; (2) literaturas do campo das ciências humanas, tais como obras de história e sociologia da alimentação, além de referenciais que trouxessem outras perspectivas de saúde, baseadas em saberes e conhecimentos tradicionais. As discussões apresentadas também resultaram de uma visão crítica das autoras norteada, sobretudo, pelas políticas em saúde e nutrição no Brasil.

\section{Desenvolvimento}

\subsection{As recomendações e práticas de saúde baseadas em evidências científicas}

Desde o início do século passado se sabe que para o bom funcionamento do corpo humano é necessário o consumo adequado de carboidratos, gorduras, proteínas, minerais e vitaminas (CANNON, 2005). Na segunda metade do século passado, o conhecimento desses componentes da dieta e seus efeitos sobre a saúde foi sendo aperfeiçoado (CANNON, 2005). O tipo de gordura, por exemplo, ganhou importância como fator de risco para doenças. Pouco se sabia sobre outros componentes da dieta e ainda nas últimas décadas do século passado, muitas substâncias contidas nos alimentos eram tomadas como fatores antinutricionais, e hoje se conhece o efeito antioxidante dessas mesmas. Surgiram os alimentos funcionais com atributos específicos de promoção da saúde. Essas substâncias dos alimentos (nutrientes ou não) têm propriedades antioxidantes, de modulação da função imune, da resposta inflamatória, entre outras (ZHAO et al., 2016). Mais recentemente, a microbiologia entrou em cena e descobriuse a importância da microbiota intestinal na modulação de funções orgânicas, incluindo o metabolismo energético (GOMEZ-LOPEZ, 2019). Para além dessas substâncias não nutritivas de importância para a saúde, numa outra direção, se percebe que o alimento não é só matéria, mas carrega consigo afeto, cultura, história e outros atributos responsáveis por saúde e bemestar. Todo esse conhecimento cientificamente acumulado precisa ser levado em conta num cenário de pandemia e ameaça à saúde. 
Mesmo que não tenham ainda sido produzidas evidências científicas específicas sobre o efeito do estado nutricional na evolução da infecção pelo novo coronavírus e recomendações frente ao enfrentamento da doença instalada (WORLD HEALTH ORGANIZATION, 2020), é importante considerar que a pandemia causada por este vírus afeta o metabolismo humano de forma multissistêmica, que pressupõe o papel do bom estado nutricional. Assim, a busca pela prevenção e pelo conhecimento deve ser incessante, considerando a complexa interação de todas as funções orgânicas em seus processos metabólicos e equilíbrio geral modulando a respostas de cada um aos fatores agressores.

Quanto às informações disponíveis sobre epidemias respiratórias no Brasil, uma das mais recentes foi a epidemia de influenza A (H1N1), ocorrida em 2009. Nesta, o perfil clínicoepidemiológico da Síndrome Respiratória Aguda Grave apresentou um destaque em relação à maior chance de evolução a óbito em indivíduos com baixa escolaridade que possuíam ao menos uma comorbidade ou com comprometimento pulmonar. O conhecimento prévio dessas informações poderia ter ajudado no planejamento do cuidado à população (FELINTO; ESCOSTEGUY; MEDRONHO, 2019). A baixa escolaridade pode estar associada à hipertensão não controlada e outros aspectos do cuidado com a saúde que a educação em saúde pode fazer a diferença.

\section{Os cuidados gerais:}

Nesse sentido, destaca-se que todos os fatores de influência são importantes, desde as boas práticas na manipulação e higiene de alimentos (WORLD HEALTH ORGANIZATION, 2020) até a troca de afeto, contribuem independentemente com a manutenção da homeostase orgânica e bem-estar, podendo ser decisivos no enfrentamento do desequilíbrio provocado pela doença (CRITTENDEN; MURPHY; COHEN, 2018).

Globalmente, as infecções respiratórias estão entre as principais causas do consumo de recursos de saúde e de morbimortalidade. Mas, em meio aos gastos com tratamentos existem recomendações que podem ser utilizadas na prevenção e promoção da saúde, tais como, as medidas gerais de vacinação antigripal, evitar o consumo de álcool e estar com o estado nutricional adequado (FROES et al., 2014).

No que diz respeito à promoção da saúde e prevenção de problemas cardiorrespiratórios, medidas simples de cuidado podem ser adotadas, como a adoção de uma alimentação equilibrada, composta por refeições nutritivas e seguras, a higiene ambiental e pessoal para a prevenção da disseminação de infecções, a desinfecção das mãos, higiene nasal, manutenção 
de ventilação no ambiente e desinfecção de objetos que são manuseados pelas crianças (MARTINS; VERÍSSIMO, 2006).

\section{Substâncias bioativas e nutrientes com propriedades funcionais/nutracêuticas}

Nos últimos tempos, as propriedades funcionais de alimentos têm despertado interesse e isso tem gerado aumento no consumo de alimentos com esses atributos (BALLUS et al., 2010). A maioria dos alimentos in natura contém uma ou mais substância bioativa, além dos nutrientes (HERNÁNDEZ-HERRERO; FRUTOS, 2014). Já, os alimentos fermentados, incluindo o leite, atuam como probióticos modulando a microbiota intestinal ao atuarem simbioticamente com vários tipos de fibras, caracterizadas como prebióticos (GALLINA et al., 2019; SHORI, 2016). Assim, a ciência tem mostrado que há uma grande variedade de nutrientes, substâncias bioativas, enzimas e probióticos com alegação de propriedades funcionais.

Entre os nutrientes com alegação funcional, estão os ácidos graxos do ômega 3 (n-3), encontrados nos peixes de água fria, nozes, frutos do mar, linhaça, orégano, cravos, pinhões, têm sido estudados como moduladores da função imune por suas propriedades antiinflamatórias (GRIMBLE, 2001; MARKOWIAK; ŚLIŻEWSKA, 2017). Além desses, existem outros nutrientes envolvidos na modulação da resposta inflamatória, entre os quais a vitamina D, a glutamina e o zinco (BERNAL CASTRO; DIAZ-MORENO; GUTIERREZ-CORTES, 2017). A vitamina D encontra-se relacionada com a massa óssea, a pele e a imunidade. Essa vitamina está presente no leite e seus derivados (HUERTAS et al., 2019). Entre 10 e $20 \%$ da vitamina $\mathrm{D}$ necessária ao organismo humano provém da dieta e por volta de $80 \%$ é sintetizada endogenamente (SARAIVA et al., 2006). No entanto, a ativação dessa vitamina em sua forma ativa, depende de luz solar. Esse é um importante aspecto a ser lembrado em tempo de confinamento social, mas será preciso que as pessoas criem estratégias para receber diariamente a luz solar (radiação ultravioleta), ao menos 20 minutos de sol ao dia, 5\% de exposição da pele (SARAIVA et al., 2006). Embora aqui se tenha dado destaque às propriedades dos ácidos graxos n-3 e da vitamina $D$, chamamos a atenção para o papel de uma dieta equilibrada que supra as necessidades de todos os nutrientes para que se garanta o bom funcionamento orgânico. Desse modo, tanto o excesso quanto a falta podem ser prejudiciais.

Embora exista evidência de que a resposta inflamatória é influenciada por fatores genéticos, a homeostase fisiológica também exerce influência e existem nutrientes que geram efeitos positivos sobre a resposta inflamatória e função imune frente às lesões e infecções 
agudas (ZHAO et al., 2016). Entre esses nutrientes estão os ácidos graxos n-3, a glutamina, a arginina, os aminoácidos sulfurados e os nucleotídeos. A suplementação desses nutrientes tem mostrado alguma melhora em pacientes críticos, mas parece que essa resposta depende do perfil genético. Nesses pacientes a modulação da função imune pode ocorrer diretamente no processo inflamatório ou indiretamente no status antioxidante (ZHAO et al., 2016). Sendo que de um lado temos a indicação da imunomodulação da resposta inflamatória aguda, de outro na inflamação crônica o efeito dos antioxidantes tem apresentado um corpo maior de evidências. No caso das doenças crônicas, a maioria envolve processos inflamatórios (doenças cardiovasculares, diabetes, obesidade, doenças reumáticas, entre outras). A inflamação gera estresse oxidativo e dano celular. Nutrientes e substâncias bioativas nos alimentos podem reduzir o estresse oxidativo e consequentemente a inflamação da doença crônica (VIDAL et al., 2012). O consumo de alimentos específicos, os quais sejam ricos em vitamina $\mathrm{C}$ e polifenóis têm sido estudados com significativos resultados: Cereja (KELLEY; ADKINS; LAUGERO, 2018), Kiwi, laranja (PAIVA, 2019).

As substâncias bioativas não entram na categoria de nutriente, mas ao constarem na dieta podem modular determinadas funções orgânicas (FREITAS; NAVES, 2010). Os polifenóis, amplamente encontrados nos vegetais, representam vários grupos de substâncias bioativas, entre os quais, estão os flavonóides, com mais de 500 compostos identificados nas frutas, hortaliças e chás, entre outros (FALLER; FIALHO, 2009). Destaque para a quercetina na cebola, a mirecitina no brócolis, as antocianinas das frutas vermelho-arroxeadas (cereja, morango, uvas) e as flavonas nas frutas cítricas (limão, laranja). As propriedades funcionais dos polifenóis estão na sua capacidade antioxidante associada à proteção cardiovascular, doenças neurodegenerativas, câncer, entre outras. Os glucosinolatos são um grupo de polifenóis responsáveis pelo sabor picante dos alimentos e são encontrados, entre outros, na canela e nos vegetais crucíferos (brócolis, couve de Bruxelas, couve flor, couve, acelga chinesas) e frutas escuras (amora, uvas vermelhas e cerejas), para os quais tem se mostrado a propriedade de reduzir a inflamação intestinal (SILVA et al., 2016). A alicina, um composto organossulfurado, encontrada no alho está na lista das substâncias bioativas com propriedades antioxidantes, entre outras (LOCATELLI et al., 2017).

Entre os alimentos podemos também citar o maracujá que em sua polpa e seus resíduos apresentam um conteúdo significativo de compostos bioativos, os quais diferem em quantidade nos tipos de "cores" de maracujá existentes, tal é a variedade e diversidade de possibilidades, reforçando a importância de uma dieta variada e diversificada (DOS REIS et al., 2018). Outro 
exemplo é a laranja que, além de nutrientes inclui em sua composição diversas substâncias bioativas, cujo consumo da fruta traz muitos benefícios à saúde. Somando-se a isso, a pectina extraída de sua casca pode ser empregada como suplemento de fibra alimentar, quanto o óleo essencial pode ter efeito antidepressivo e sedativo, efeito antioxidante e germicida, utilizado como suplemento alimentar ou até ser como princípio ativo em medicamentos (YWASSAKI; CANNIATTI-BRAZACA, 2011).

As fibras alimentares (BERNAUD; RODRIGUES, 2013) são de vários tipos e distribuem-se em vários grupos a depender da sua composição. São carboidratos resistentes às enzimas digestivas que, no entanto, além de modular o peristaltismo intestinal, sofrem ação de bactérias do cólon gerando metabólitos com importantes atividades biológicas. Os efeitos protetores das fibras têm sido relacionados às doenças cardiovasculares, hipertensão arterial sistêmica, diabetes, desordens gastrointestinais, melhora da função imune e controle do peso corporal (BERNAUD; RODRIGUES, 2013). Entre as fibras estão os frutanos, representados principalmente pela inulina e pelos frutooligossacarídeos (FOS) (PIMENTEL; GARCIA; PRUDENCIO, 2012). Uma das principais fontes de inulina é a raiz da chicória, mas existem muitas outras fontes, tais como: trigo, beterraba, alho poró, aspargo, cebola, banana e banana da terra (MACEDO; VIMERCATI; ARAÚJO, 2020). As fibras alimentares são importantes substratos para as bactérias do cólon, as quais atuam como prebióticos.

A modificação da trajetória da doença por meio de dieta e probióticos tem ganho destaque nas pesquisas científicas, envolvendo a microbiota e seus metabólitos na restauração do equilíbrio normal metabólico, considerando o seu caráter de funcionamento multissistêmico (MORENO DEL CASTILLO; VALLADARES-GARCIA; HALABE-CHEREM, 2018). O principal composto envolvido como metabólito resultante da ação das bactérias sobre as fibras vegetais é o butirato, um ácido graxo de cadeia curta com importantes funções fisiológicas (MARKOWIAK; ŚLIŻEWSKA, 2017; SANCHEZ; PANAHL; TREMBLAY, 2015).

A modulação da microbiota intestinal por meio dos probióticos dietéticos envolve a resposta inflamatória, em especial a inflamação das doenças crônicas (VALENTINI et al., 2020). O comprometimento na microbiota intestinal é chamado de disbiose e atualmente foi associado como um fator modulador da obesidade (ANDRADE et al., 2015). Esse desequilíbrio pode ocorrer devido ao abuso de certos medicamentos, como antibióticos e inibidores da bomba de prótons, entre outros (MOLONEY et al., 2014). A relação entre o microbioma e doenças humanas derivam da perda de funções do cólon pela presença de microorganismos patogênicos em detrimento daqueles favoráveis (GOMEZ-LOPEZ, 2019). Por isso, é importante o equilíbrio 
da microbiota, sendo uma prioridade para manter a saúde integral do indivíduo (HARO et al., 2016). Relativo à proteção da função pulmonar, podemos especular que o entendimento da interação sistêmica com microbioma pulmonar e o seu eixo intestino-pulmão que ocorre diretamente pela microrrespiração pode estar envolvido na modulação da resposta imune proporcionando evolução no tratamento e prevenção de doenças (COSTA et al., 2018). Os alimentos derivados do leite tem sido o principal veículo de bactérias benéficas para o cólon, mas existem outros produtos fermentados como: chucrute, o kefir de coco, a kombucha e o gengibre em conserva que estão relacionados à proteção ao sistema imune e resposta inflamatória (SANTOS, 2011).

Portanto, dada a situação de isolamento social e quarentena, a alimentação equilibrada é estratégica para a saúde e depende de cada um no cuidado de si, mas também de ações coletivas que devem ser planejadas e executadas no âmbito local, regional e nacional visando a saúde e bem-estar da população brasileira (BRASIL, 2020b).

O número de compostos bioativos é extremamente grande, o que nos leva a refletir sobre a vantagem de consumi-los nos alimentos, mesmo frente a sua concentração quando fornecidos pelos suplementos alimentares. Em relação aos nutrientes, a suplementação também deve ser adotada com parcimônia visto que, em várias situações o desequilíbrio na oferta pode gerar também desequilíbrio metabólico. Os profissionais da saúde, nutricionistas de um modo particular devem ter uma boa formação de bioquímica nutricional e manter-se atualizados para prescrever os suplementos de forma adequada. E, melhor seria, se eles só pudessem ser adquiridos e consumidos se devidamente orientados. $\mathrm{Na}$ alimentação, quem não conhece bioquímica da nutrição não vai errar se consumir vegetais das mais variadas cores e partes das plantas, incluindo as sementes e oleaginosas.

Como vimos, as alegações de propriedades funcionais (ou nutracêuticas) são muitas, de um modo geral, as vitaminas e carotenóides têm efeitos protetores nas doenças cardiovasculares; determinados ácidos graxos estão relacionados a efeitos protetores às doenças cardiovasculares, sistema imune e ação antioxidante, protegendo diversos tecidos orgânicos; as fibras alimentares têm sido associadas à proteção cardiovascular, carcinogênese e função intestinal; os flavonóides tem ação anti-oxidantes e os probióticos promovendo a modulação e geração de metabólitos pela flora intestinal, em especial a produção de butirato. Algumas plantas têm efeitos hipoglicemiantes (SILVA et al., 2016).

A regulação dos suplementos alimentares em termos de composição e divulgação no Brasil é feita pela Agência Nacional de Vigilância Sanitária (ANVISA), assim como é feita 
também a regulação da "alegação de saúde" nos rótulos dos alimentos. A Resolução da Diretoria Colegiada (RDC 243 de julho de 2018) ANVISA estabelece listas de constituintes, de limites de uso, de alegações e de rotulagem complementar dos suplementos alimentares (BRASIL, 2018b). O quadro 1 apresenta alguns nutrientes de interesse (na resolução constam macronutrientes, minerais e vitaminas) e as substâncias bioativas permitidas nos suplementos alimentares, bem como as alegações autorizadas (que não ocorre para todos os alimentos), elaboradas a partir de evidências científicas comprovadas. Lembrando que quando procedimentos são realizados industrialmente para isolar ou condensar essas substâncias temos como resultado os nutracêuticos. De maneira geral, os suplementos, apresentam os princípios ativos de forma concentrada e a ANVISA também regula os valores mínimos e máximos nesses produtos (BRASIL, 2018b).

Relativos aos ácidos graxos n-3, a alegação de propriedade funcional autorizada pela ANVISA é: “Os ácidos graxos ômega 3 (n-3) EPA e DHA auxiliam na redução dos triglicerídeos", enquanto não há referência para alegações relativas ao ácido graxo linolênico (n-3) ou linoléico (n-6) das fontes vegetais, mesmo que se tenha indicação de valores mínimos nos suplementos (BRASIL, 2018b). Lembrando que a normativa se refere aos suplementos alimentares e não aos alimentos em si.

Quadro 1. Nutrientes selecionados e substâncias bioativas componentes de suplementos alimentares (BRASIL, 2018a).

\begin{tabular}{|c|c|}
\hline Nutrientes & Alegação \\
\hline \multirow{3}{*}{ Fibras } & $\begin{array}{l}\text { As fibras alimentares auxiliam no funcionamento } \\
\text { do intestino }\end{array}$ \\
\hline & $\begin{array}{l}\text { O psyllium auxilia na redução do colesterol } \\
\text { sanguíneo }\end{array}$ \\
\hline & $\begin{array}{l}\text { A quitosana auxilia na manutenção dos níveis de } \\
\text { colesterol sanguíneo }\end{array}$ \\
\hline Ácidos graxos EPA e DHA & $\begin{array}{l}\text { Fonte de ômega } 3 \\
\text { Os ácidos graxos ômega } 3 \text { EPA e DHA auxiliam } \\
\text { na redução dos triglicerídeos. }\end{array}$ \\
\hline Ácido graxo linolênico n-3 & - \\
\hline Ácido graxo linoléico n-6 & - \\
\hline Substâncias bioativas & - \\
\hline 10-HDA* (da geléia real) & - \\
\hline
\end{tabular}




\begin{tabular}{|l|c|}
\hline Ácido Clorogênico (do café) & - \\
\hline Alicina (do alho) & - \\
\hline Astaxantina (carotenóide) & \multicolumn{1}{|c|}{-} \\
\hline Cafeína (incluindo guaraná em pó) & \multicolumn{1}{|c|}{-} \\
\hline Coenzima Q10 & Auxiliam na redução da absorção do colesterol \\
\hline Compostos fenólicos (incluindo própolis) & $\begin{array}{l}\text { "Nos produtos adicionados de probióticos, o } \\
\text { benefício deve ser comunicado por meio da } \\
\text { alegação de propriedade funcional ou de saúde } \\
\text { aprovada para a linhagem, exceto quando houver } \\
\text { disposição em contrário em regulamento técnico } \\
\text { específico" } \\
\text { Resolução RDC241/2018 (BRASIL, 2018a) }\end{array}$ \\
\hline Fosfatidilserina & \multicolumn{1}{|c|}{} \\
\hline Probióticos &
\end{tabular}

* Ácido 10-hidróxi-2- decenóico

Fonte dos dados: Agência Nacional de Vigilância Sanitária (BRASIL, 2018a)

Em relação aos alimentos, no Brasil, a legislação vigente (RDC-360/2003) data de 2003 (BRASIL, 2003). Esse Regulamento Técnico para rotulagem de alimentos indica apenas que facultada a indicação de propriedades nutricionais. Não é permitida a "indicação de propriedades medicinais ou terapêuticas ou aconselhar o seu consumo como estimulante, para melhorar a saúde, prevenir doenças ou com ação curativa" (BRASIL, 2008, p.8). Em 2006 o Ministério da Saúde publicou um amplo estudo sobre informação nutricional e alegação de saúde em 77 países (BRASIL, 2006b) e, em 2019, foi submetida à consulta pública uma nova lei de rotulagem. Na proposta, as alegações para os ácidos graxos (n-3, n-6 e n-9) aparecem como "fonte de" e "alto conteúdo", enquanto as fibras, soma-se além dessas, a alegação “aumentado" e não permite especificar o tipo de fibra (BRASIL, 2019).

\section{A Atividade Física:}

A importância da atividade física para a saúde é reconhecida pela ciência. Esta se torna ainda mais importante, sobretudo, no contexto da COVID-19 devido ao auxílio na resposta imunológica, controle de doenças, melhoria da capacidade funcional e saúde mental (RAIOL, 2020). Porém, o distanciamento social, que obriga as pessoas a permanecerem em casa, como 
importante estratégia no combate a pandemia, pode levar a diminuições drásticas nos níveis de atividade física.

Em relação à influência das atividades físicas na função pulmonar, existem dados de pesquisas que mostram que a prática rotineira de exercício físico preserva a função pulmonar e pode estar relacionada à longevidade (LUZAK et al., 2017). Isso indica que o incentivo a prática de exercícios é necessário, mesmo que seja em ambiente domiciliar. A manutenção de exercícios físicos ao longo da vida preserva todos os sistemas orgânicos (VALENZUELA et al., 2020). Confirmando o que já foi dito, Fuertes et al. (2018) mostraram que atividades físicas no lazer foi associada à qualidade da função respiratória (FUERTES, 2018). A intensidade do exercício físico relaciona-se com o desenvolvimento da capacidade cardiorrespiratória. $\mathrm{O}$ exercício físico também tem sido relacionado com a resposta inflamatória junto à função pulmonar (STIES et al., 2018). Embora até o momento não se tenha resultados de estudos que mostram que um melhor condicionamento físico e função pulmonar evitam as complicações da COVID-19, um pulmão debilitado certamente terá mais dificuldade para enfrentar o problema.

A inflamação crônica associa-se positivamente ao índice de massa corporal (IMC). Os principais fatores associados ao excesso de peso são a alimentação inadequada, a inatividade física e o estresse da vida diária (SKARPSNO et al., 2019). Foi mostrado que o estado inflamatório presente no excesso de peso pode estar associado ao estresse e insônia e fadiga mental (SKARPSNO et al., 2020).

Assim, no isolamento social o exercício físico ganha importância na preservação da função pulmonar e no controle do peso corporal. Estando mais tempo em casa, a tendência das pessoas é comer com mais frequência e tornam-se propensas ao ganho de peso corporal. Enquanto, para as crianças, diferentes brinquedos de sopro podem ter efeito potencial na mecânica respiratória de crianças saudáveis. Pois, a soma dos estímulos ventilatórios reduz a resistência das vias aéreas (SCHIVINSKI et al., 2020). Os exercícios respiratórios também deveriam ser feitos por adultos e principalmente por idosos.

\section{A saúde ambiental:}

Exercícios ao ar livre, contato com a natureza e ar puro são indicadores de saúde. A natureza é gratuita e, ao menos teoricamente, todos nós podemos usufruir desse benefício. Por outro lado, os ambientes insalubres afetam a saúde e por suposto a saúde respiratória. A insalubridade ambiental pode ser determinada pelas iniquidades sociais, constituindo-se num problema de saúde pública. No entanto, o ambiente doméstico pode ser insalubre também por 
falhas na higiene e ventilação. Pessoas confinadas em casa, algumas vezes não acostumadas à realização das tarefas domésticas podem não se dar conta desses cuidados. A proliferação de fungos em ambientes pouco arejados, a umidade e a poeira relacionam-se com complicações respiratórias (ARAÚJO-MARTINS et al., 2014). No mínimo, esse cuidado deve ser tomado para que não se necessite de cuidados médicos hospitalares, num momento tão delicado quanto ao que estamos vivendo.

\section{O cuidado integral:}

O cuidado deve ser tomado como elemento central frente à pandemia da COVID-19, seja ele individual e coletivo. A saúde deve ser priorizada e uma abordagem holística parece ser a mais conveniente na atual conjuntura. Há que se cuidar do corpo, da mente e do entorno (físico e social). Defendemos essa abordagem e consideramos que os profissionais de saúde, em equipe, devem empenhar-se nessa linha de atuação, pois na abordagem holística que se relaciona às diversas manifestações físicas e psicossociais. Segundo Sampaio (2019), essa abordagem na saúde, tanto na prevenção, quanto no tratamento das doenças, proporciona: o aumento da tolerância à prática de exercícios físicos, disposição para as atividades diárias, autonomia, adesão à terapia, melhor qualidade de vida e bem-estar, além da diminuição da dispnéia e da ansiedade. Estas melhorias foram proporcionadas por meio da constância de uma programação com aprendizado de técnicas respiratórias, intervenção nutricional, psicossocial, treino de exercícios e atividades diárias (SAMPAIO, 2019).

Uma abordagem integral abarca um importante componente da saúde, que transcende o conhecimento cientificamente produzido, como o discutido até aqui e traz para a discussão dos conhecimentos tradicionais e outros elementos culturais de extrema importância.

\subsection{Saberes e conhecimentos tradicionais}

Sentimos insegurança, uma paranoia da queda porque as outras possibilidades que se abrem exigem implodir essa casa que herdamos, que confortavelmente carregamos em grande estilo, mas passamos o tempo inteiro morrendo de medo. Então, talvez o que a gente tenha de fazer é descobrir um paraquedas. Não eliminar a queda, mas inventar e fabricar milhares de paraquedas[...]. Então, que a gente pare de despistar essa nossa vocação e, em vez de ficar inventando outras parábolas, que a gente se renda a essa principal e não se deixe iludir com o aparato da técnica. $\mathrm{Na}$ verdade, a ciência inteira vive subjugada por essa coisa que é a técnica. (KRENAK, 2019, p. 31)

A citação que acabamos de enunciar, proferida pelo líder indígena Ailton Krenak (2019), expressa a concepção indígena a respeito da tecnologia. Essa concepção é pertinente 
neste trabalho por questionar um modelo único de se compreender a ciência, voltado principalmente para a produção de técnicas e tecnologias. Portanto, ainda que a importância desta seja inquestionável para a produção da vacina para a COVID-19, por exemplo, não deixa de ser importante a reflexão de Krenak. Também é necessário questionar o conhecimento validado cientificamente como único modelo de saber, sendo importante desenvolver e valorizar outras modalidades de conhecimento, e neste caso, outras formas de se compreender os saberes relativos à saúde. Assim, outros paradigmas de saúde e outros paradigmas científicos podem atuar como "paraquedas" neste momento, amenizando as múltiplas consequências da pandemia. Busca-se, portanto, apresentar brevemente práticas de saúde e concepções alimentares baseadas em saberes e conhecimentos tradicionais.

\section{Terapias:}

Uma vez que a COVID-19 não afeta exclusivamente a saúde dos infectados, mas também modifica as condições de saúde da população de forma geral, devido às mudanças provocadas pelo isolamento social, apresentamos estratégias para a manutenção e promoção da saúde. Nesse sentido, para além das evidências científicas da saúde, procuramos destacar alguns conhecimentos e terapias tradicionais pertinentes ao contexto da pandemia. Para isso, buscamos apresentar algumas dessas terapias, sobretudo, com base nas terapias expostas pelas diretrizes da Política Nacional de Práticas Integrativas e Complementares no SUS (PNPIC). Esta política tem por base o "modelo de atenção humanizada e centrada no indivíduo" (p. 4-5) e que considera "o indivíduo na sua dimensão global - sem perder de vista sua singularidade, quando da explicação de seus processos de adoecimento e saúde" (BRASIL, 2006a, p. 5). A abordagem defendida pela PNPIC é essencial, sobretudo neste contexto, pois parte do pressuposto de que este modelo de trabalhar a saúde amplia a corresponsabilização dos indivíduos por sua saúde, contribuindo assim para o aumento do exercício da cidadania. Assim, para este momento é importante fortalecer abordagens que visem "estimular os mecanismos naturais de prevenção de agravos e recuperação da saúde por meio de tecnologias eficazes e seguras [...]. Outros pontos compartilhados pelas diversas abordagens abrangidas neste campo são a visão ampliada do processo saúde-doença e a promoção global do cuidado humano, especialmente do autocuidado" (BRASIL, 2006a, p. 10).

Uma das práticas terapêuticas mais reconhecidas é a Medicina Tradicional Chinesa, sua expansão é um dos objetivos da Organização Mundial da Saúde (OMS). Esta caracterizase por um sistema médico integral, ou seja, sua linguagem retrata simbolicamente as leis da 
natureza e que valoriza a inter-relação harmônica entre as partes, visando a integridade (BRASIL, 2006a). Fundamenta-se em teorias como o Yin-Yang, visando o equilíbrio da dualidade.

Outra terapêutica recomendada pela PNPIC é a fitoterapia, esta é caracterizada pelo uso de plantas medicinais de diferentes formas farmacêuticas, sem a utilização de substâncias ativas isoladas (BRASIL, 2006a). Ela tem sido enfatizada em diferentes estudos por trazer resultado na redução de níveis de estresse, ansiedade e melhoria de qualidade de vida. Assim, "O uso de plantas medicinais na arte de curar é uma forma de tratamento de origens muito antigas, relacionada aos primórdios da medicina e fundamentada no acúmulo de informações por sucessivas gerações" (BRASIL, 2006a, p. 19).

Cientificamente essas terapêuticas também passam a ser cada vez mais reconhecidas. Em um estudo realizado em 2015 na cidade de São Paulo no Brasil foram avaliados adultos saudáveis atendidos no Instituto de Terapia Integrada e Oriental, monitorados por três semanas pela Lista de Sintomas de Estresse (SSL), Ansiedade Inventário-Traço e Estado quanto à qualidade de vida. Os grupos aleatorizados receberam a intervenção com frasco de fórmula Gan May Zao (GMDZ) com $50 \mathrm{ml}$ ou placebo, por fim a fórmula GMDZ reduziu os níveis de estresse (KUREBAYASHI et al., 2016).

Assim, tais terapias já reconhecidas por suas importâncias culturais, passam a ser cada vez mais reconhecidas também cientificamente. Outra terapia que passa a ser mais utilizada é o Reiki, classificada como energética de biocampo promove benefícios na dor, ansiedade / depressão e qualidade de vida dos pacientes em várias condições (BILLOT et al., 2019). O Reiki é uma terapia de 2500 anos conhecida por ser energética, vibracional ou sutil, com ação facilitada por um leve toque no corpo ou acima dele, é uma ótima alternativa no tratamento dessas doenças (JOYCE; HERBISON, 2015), pois os tratamentos da ansiedade e da depressão costumam utilizar medicamentos, isto altera o metabolismo, o que não é interessante, sobretudo neste contexto em que a manutenção da imunidade deve ser uma preocupação central. Outro ponto negativo no uso de medicamentos é que muitos causam dependência devido ao tempo necessário de uso e efeitos adversos.

\section{Alimentação: tradição e cultura:}

Assim, como discutimos, a alimentação é essencial no aspecto nutricional, contudo, também é importante por determinados alimentos desempenharem funções terapêuticas, por exemplo, como é o caso da fitoterapia. Em outra dimensão, porém, a alimentação possui grande 
relevância em seus aspectos culturais, refletindo a cultura, tradições e identidades. De acordo com Contreras e Gracia (2011, p. 29) cultura alimentar é "o conjunto de representações, crenças, conhecimentos e práticas herdadas e/ou aprendidas que estão associadas à alimentação e são compartilhadas pelos indivíduos de uma dada cultura ou de um grupo social determinado".

Como exemplo de conhecimentos transmitidos através da cultura alimentar, citamos as especiarias, sendo principalmente por suas funções terapêuticas que estes produtos se difundiram e marcaram historicamente os séculos XIV, XV e XVI. Segundo Flandrin e Montanari (2018), as especiarias se destacaram em ao menos três diferentes funções: conservação, distinção social e difusão da cozinha árabe. O autor aponta que "Muitos desses produtos importados do Oriente não tinham uma função culinária, mas terapêutica. Quando àqueles usados pelos cozinheiros, também eles eram usados como medicamento" (FLANDRIN; MONTANARI, 2018, p. 480). Os livros desse período apresentavam sobretudo suas funções terapêuticas, afirmando que as especiarias "num primeiro momento importantes como medicamento e só depois para temperar os alimentos" (FLANDRIN; MONTANARI, 2018, p. 481). Assim, "A questão importante, uma vez que a maioria das drogas das quais abusamos, contra as advertências dos médicos, inicialmente foram usadas por sugestões deles, por motivos médicos. É o caso, por exemplo, do açúcar, do café, do chá, do tabaco, do álcool, etc.” (p. 481). É certo que no caso das especiarias, do século XIV até o momento atual, muito foi transformado no que se refere à alimentação e consequentemente nos significados das especiarias. Porém, sua importância em preparações culinárias e como tradições terapêuticas, curativas e preventivas, são grandes ferramentas na prevenção de doenças e ilustram como a cultura alimentar ultrapassa barreiras dos saberes, tomando há séculos proporções mundiais.

Em outro exemplo, na cultura indígena, temos alguns alimentos que expressam a importância nutricional e cultural da alimentação, tais como: milho, mandioca, banana, amendoim, cana, batata-doce, abacaxi e mel. Porém, o consumo destes por esses povos estão ameaçados pelo contato com a civilização urbana que levou essa população a consumir alimentos industrializados, essa mudança além de alterar o estado nutricional, também resultou em "novas" doenças, as crônicas (MOURA; BATISTA; MOREIRA, 2010). Neste caso temos um duplo ataque: à saúde indígena, que se fragiliza com a inserção desses alimentos, e também um ataque cultural, que leva a um epistemicídio ${ }^{2}$ desta.

\footnotetext{
${ }^{2}$ De acordo com Boaventura de Souza Santos em Epistemologias do Sul, epistemicídio consiste na supressão dos conhecimentos locais perpetrados por um conhecimento alienígena, ou seja, o assassinato do conhecimento da própria cultura subordinada a conhecimentos de outros grupos sociais, cuja maior representação é o próprio colonialismo europeu.
} 
Um momento como este que estamos vivendo, nos leva a voltar nossos olhares para a aventura humana aqui na terra, a nossa civilização e o quanto os alimentos estiveram presentes, em todas as suas dimensões. Leva-nos a refletir sobre o quanto se produziu e se acumulou de conhecimentos sobre a relação saúde e alimento e sobre o rico arsenal biocultural que dispomos, além daquela que já perdemos. Cores e sabores, variedade e diversidade das culturas alimentares geram bem-estar da dimensão da comensalidade. Para a ciência, pigmentos, óleos essenciais, substâncias bioativas, respostas diversas aos estímulos das papilas gustativas e modulação das funções orgânicas. Desde sempre estamos falando dos mesmos alimentos que alimentam o corpo e o espírito, gerando saúde e bem-estar.

\subsection{Integrando os saberes}

Assim, este trabalho se propôs a discutir diferentes fontes de saberes para pensar a saúde e nutrição frente à COVID-19. Reforçamos a importância da alimentação saudável tal como ela é concebida hoje com base no mais atualizado conhecimento científico. A ciência tem revelado continuamente as propriedades dos alimentos, mostrando cada vez mais a complexidade desses mesmos, muito além dos nutrientes conhecidos. Tem revelado substâncias bioativas inclusive em partes que não tem sido aproveitada dos alimentos (YWASSAKI; CANNIATTIBRAZACA, 2011; STORCK et al., 2013). Essas descobertas podem representar um grande incentivo para o exercício culinário, valorizando a diversidade e variedade dos alimentos saudáveis frescos e seguros, os alimentos que se encontram com os produtores locais, hortas, feiras e mercadinhos. No entanto, atendendo o interesse do grande mercado, nossas farmácias estão lotadas de suplementos alimentares. Não queremos dizer com isso que os suplementos não tenham a sua utilidade e indicação. Apenas queremos colocar em destaque o que as evidências científicas têm mostrado: os alimentos in natura são insubstituíveis.

Por outra perspectiva, os alimentos devem estar de acordo com seu contexto cultural. Segundo o Guia Alimentar para a População Brasileira (BRASIL, 2014, p. 15):

Alimentação diz respeito à ingestão de nutrientes, mas também aos alimentos
que contêm e fornecem os nutrientes, a como alimentos são combinados entre
si e preparados, a características do modo de comer e às dimensões culturais
e sociais das práticas alimentares. Todos esses aspectos influenciam a saúde e
o bem-estar.

Nesse sentido, uma alimentação adequada pressupõe também o “"reconhecimento” e o 'conhecimento' das diferenças culturais, do papel desempenhado pela socialização no consumo de alimentos e das implicações sociais da comida para a saúde e para o ambiente" (CONTRERAS; GRACIA, 2011, p. 16). Por isso, a atenção na escolha dos alimentos é tão 
importante. Esta deve levar em conta, inclusive a regionalidade e a sazonalidade, pois afetam até mesmo a qualidade do alimento.

De acordo com Pareda (2019), os produtos locais são mais ricos em nutrientes, como foi observado nos frutos de Physalis peruviana L. que é uma boa fonte de nutrientes e compostos bioativos e quando foram produzidos em outra região tiveram propriedades nutricionais e os compostos bioativos (vitamina $\mathrm{C}$ e $\beta$-caroteno) foram diferentes, fatores que podem ser atribuídos a condições ecológicas não adequadas (BAZALAR PEREDA; NAZARENO; VITURRO, 2019). A questão regional também deve ser observada no âmbito da fitoterapia, visto que "O Brasil possui grande potencial para o desenvolvimento dessa terapêutica, como a maior diversidade vegetal do mundo, ampla sociodiversidade, uso de plantas medicinais vinculado ao conhecimento tradicional e tecnologia para validar cientificamente este conhecimento" (BRASIL, 2006a, p. 19).

O problema é que mesmo com tantos conhecimentos e riquezas culturais na alimentação e nos hábitos, os perfis multidimensionais de fatores de risco são relacionados ao estilo de vida brasileiro (PEREIRA; GONZAGA; LYRA, 2019). Há a necessidade de pensar nos aspectos físicos, psicológicos e comportamentais associados, considerando os aspectos sociais, econômicos, culturais e espirituais individuais. Infelizmente a sociedade ocidental é predominante sedentária, além de possuir uma ingestão desequilibrada do consumo de alimentos, isso afeta a saúde. Visto que as evidências científicas mostram a correlação positiva da prática de atividade física e alimentação saudável com uma melhor qualidade de vida e prevenção de doenças (CHALES-AOUN; MENINO, 2019). Ressaltamos assim a prática de hábitos culinários conectados a produção de alimentos e seus significados. Trazendo assim "Os dez passos para uma alimentação adequada e saudável" (BRASIL, 2014), como um norteador no contexto da pandemia. Finalizamos na concordância de Bezerra $(2018$, p. 112) de que é preciso:

[...] Ultrapassar os limites de saberes específicos, altamente especializados. Implica correr os riscos que podem vir do trânsito por outros espaços disciplinares em busca do diálogo e de abertura de fendas nas cercas e muros que separam campos de conhecimento e construir novas posturas profissionais e intelectuais frente à complexidade de fenômenos alimentares, cuja natureza não se aparta da complexidade da vida. (BEZERRA, 2018, p. 112). 


\section{Referências}

ANDRADE, V. L. A. et al. Obesidade e microbiota intestinal. Rev. Med. Minas Gerais, Belo Horizonte, v. 25, n. 4, p. 583-589, 2015. DOI 10.5935/2238-3182.20150126.

ARAÚJO-MARTINS, J. et al. Environment and Health in Children Day Care Centres (ENVIRH) - Study rationale and protocol. Rev. Port. Pneumol., Lisboa, v. 20, n. 6, p. 311323, 2014. DOI 10.1016/j.rppneu.2014.02.006.

BALLUS, C. A. et al. Aspectos científicos e tecnológicos do emprego de culturas probióticas na elaboração de produtos lácteos fermentados: revisão. Bol. CEPPA, Paraná, v. 28, n. 1, p. 85-96, 2010. DOI 10.5380/cep.v28i1.17900.

BAZALAR PEREDA, M. S.; NAZARENO, M. A.; VITURRO, C. I. Nutritional and antioxidant properties of Physalis Peruviana 1. fruits from the Argentinean Northern Andean Region. Plant Foods Hum. Nutr., Dordrecht, v. 74, n. 1, p. 68-75, 2019. DOI 10.1007/s11130-018-0702-1.

BERNAL CASTRO, C. A.; DIAZ-MORENO, C.; GUTIERREZ-CORTES, C. Probióticos y prebióticos en matrices de origen vegetal: avances en el desarrollo de bebidas de frutas. Rev. Chil. Nutr., Santiago, v. 44, n. 4, p. 383-392, 2017. DOI 10.4067/s071775182017000400383 .

BERNAUD, F. S. R.; RODRIGUES, T. C. Fibra alimentar: ingestão adequada e efeitos sobre a saúde do metabolismo. Arq. Bras. Endocrinol. Metab., São Paulo, v. 6, n. 57, p. 397-405, 2013.

BEZERRA, J. A. B. Educação alimentar e nutricional: articulação de saberes. Brasília, DF: FNDE, 2018.

BILLOT, M. et al. Reiki therapy for pain, anxiety and quality of life. BMJ Support. Palliat. Care, London, v. 9, n. 4, p. 434-438, 2019. DOI: 10.1136 / bmjspcare-2019-001775.

BRASIL. Agência Nacional de Vigilância Sanitária. Consulta pública n 708, de 13 de setembro de 2019. Brasília: ANVISA, 2019. Disponível em: http://portal.anvisa.gov.br/documents/10181/3882585/SEI_ANVISA+-+0734894++ Consulta+P\%C3\%BAblica+708.pdf/60eb27d4-a2c7-4c04-bbc3-08927fb4d85b. Acesso em: 07 set. 2020.

BRASIL. Agência Nacional de Vigilância Sanitária. Ministério da Saúde. Rotulagem nutricional obrigatória: manual de orientação aos consumidores. Brasília: Ministério da Saúde, 2008.

BRASIL. Agência Nacional de Vigilância Sanitária. Resolução RDC no 241, de 27 de julho de 2018. Dispõe sobre os requisitos para comprovação da segurança e dos benefícios à saúde dos probióticos para uso em alimentos. Brasília: ANVISA, 2018a.

BRASIL. Ministério da Saúde. Agência Nacional de Vigilância Sanitária. Resolução RDC n 360, de 23 de dezembro de 2003. Brasília: ANVISA, 2003.

BRASIL. Ministério da Saúde. Guia alimentar para a população brasileira. Brasília: Ministério da Saúde, 2014. Disponível em:

http://portalarquivos.saude.gov.br/images/pdf/2014/novembro/05/Guia-Alimentar-para-a-popbrasiliera-Miolo-PDF-Internet.pdf. Acesso em: 07 set. 2020. 
BRASIL. Ministério da Saúde. Instrução normativa $n^{\circ} 28$ de 26 de julho de 2018. Estabelece as listas de constituintes, de limites de uso, de alegações e de rotulagem complementar dos suplementos alimentares. Diário Oficial da União: seção 1, Brasília, DF, ano 144, n. 28, p. 130, 27 jul. $2018 b$.

BRASIL. Ministério da Saúde. Orientações sobre a prática de atividade física durante o período de quarentena. Brasília: Ministério da Saúde, 2020a.

BRASIL. Ministério da Saúde. Recomendações de alimentação e Covid-19. Brasília: Ministério da Saúde, 2020b.

BRASIL. Ministério da Saúde. Secretaria de Atenção à Saúde. Departamento de Atenção Básica. Política Nacional de Práticas Integrativas e Complementares no SUS - PNPICSUS. Brasília: Ministério da Saúde, 2006a. Disponível em: https://bvsms.saude.gov.br/bvs/publicacoes/pnpic.pdf. Acesso em: 07 set. 2020.

BRASIL. Organização Pan-Americana da Saúde. Agência Nacional de Vigilância Sanitária. Informação Nutricional e Alegações de Saúde: o cenário global das regulamentações. Brasília: OPAS, ANVISA, 2006b.

CANNON, G. The rise and fall of dietetics and of nutrition science, $4000 \mathrm{BCE}-2000 \mathrm{CE}$.

Public Health Nutr., Wallingford, v. 8, n. 6, p. 701-705, 2005. DOI 10.1079/phn2005766.

CHALES-AOUN, A. G.; MENINO, J. M. E. Actividad física y alimentación en estudiantes universitarios chilenos. Cienc. Enferm., Concepción, v. 25, n. 16, p. 28, 2019. DOI 10.4067/s0717-95532019000100212.

CONTRERAS, J.; GRACIA, M. Alimentação, sociedade e cultura. 22. ed. Rio de Janeiro: Editora Fiocruz, 2011. Tradução: Mayra Fonseca e Barbara Atie Guidalli.

COSTA, A. N. et al. O microbioma pulmonar: desafios de um novo paradigma. J. Bras. Pneumol., São Paulo, v. 44, n. 5, p. 424-432, 2018. DOI 10.1590/s180637562017000000209.

CRITTENDEN, C. N.; MURPHY, M. L. M.; COHEN, S. Social integration and age-related decline in lung function. Health Psychol., Washington, v. 37, n. 5, p. 472-480, 2018. DOI $10.1037 /$ hea0000592.

DOS REIS, L. C. R. et al. Antioxidant potential and physicochemical characterization of yellow, purple and orange passion fruit. J. Food Sci. Technol., Campinas, v. 55, n. 7, p. 2679-2691, 2018. DOI 10.1007/s13197-018-3190-2.

FALLER, A. L. K.; FIALHO, E. Disponibilidade de polifenóis em frutas e hortaliças consumidas no Brasil. Rev. Saúde Pública, São Paulo, v. 43, n. 2, p. 211-218, 2009. DOI 10.1590/S0034-89102009005000010.

FELINTO, G. M.; ESCOSTEGUY, C. C.; MEDRONHO, R. A. Fatores associados ao óbito dos casos graves de influenza A (H1N1) pdm09. Rev. Saúde Colet., Rio de Janeiro, v. 27, n. 1, p. 11-9, 2019. DOI 10.1590/1414-462x201900010433.

FLANDRIN, J-L.; MONTANARI, M. História da alimentação. 9. ed. São Paulo: Estação Liberdade, 2018.

FREITAS, J. B.; NAVES, M. M. V. Composição química de nozes e sementes comestíveis e sua relação com a nutrição e saúde. Rev. Nutr., Campinas, v. 23, n. 2, p. 269-279, 2010. 
FROES, F. et al. Consensus document for the prevention of respiratory infections in adults. Rev. Port. Pneumol., Lisboa, v. 20, n. 2, p. 111-114, e 2014. DOI 10.1016/j.rppneu.2014.02.001.

FUERTES, E. et al. Leisure-time vigorous physical activity is associated with better lung function: the prospective ECRHS Study. Thorax, London, v. 73, n. 4, p. 376-384, 2018. DOI 10.1136/thoraxjnl-2017-210947.

GALLINA, D. A. et al. Desenvolvimento e caracterização de smoothies fermentados com probióticos. Rev. Ciênc. Agron., Fortaleza, v. 50, n. 3, p. 378-386, 2019. DOI 10.5935/18066690.20190045 .

GOMEZ-LOPEZ, A. Microbioma, salud y enfermedad: probióticos, prebióticos y simbióticos. Biomédica, Bogotá, v. 39, n. 4, p. 617-621, 2019.

GRIMBLE, R. F. Nutritional modulation of immune function. Proc. Nutr. Soc., London, v. 60, n. 3, p. 389-397, 2001. DOI 10.1079/pns2001102.

HARO, C. et al. Intestinal microbiota is influenced by gender and body mass index. PLoS One, San Francisco, v. 11, n. 5, p. 154-190, 2016. DOI 10.1371/journal.pone.0154090.

HERNÁNDEZ-HERRERO, J. A.; FRUTOS, M. J. Effect of concentrated plum juice on physicochemical and sensory properties of yoghurt made at bench top scale. Int. J. Dairy Technol., Huntingdon, v. 67, n. 1, p. 123-128, 2014. DOI 10.1111/1471-0307.12101.

HUERTAS, J. R. et al. Leche y productos lácteos como vehículos de calcio y vitamina D: papel de las leches enriquecidas. Nutr. Hosp., Madri, v. 36, n. 4, p. 962-973, 2019.

JOYCE, J.; HERBISON, G. P. Reiki for depression and anxiety. Cochrane Database Syst. Rev., Oxford, v. 4, n. 1, p. 1-30, 2015. DOI 10.1002/14651858.CD006833.

KELLEY, D. S.; ADKINS, Y.; LAUGERO, K. D. A review of the health benefits of cherries. Nutrients, Basel, v. 10, n. 3. p. 368, 2018. DOI 10.3390 / nu10030368.

KRENAK, A. Ideias para adiar o fim do mundo. São Paulo: Companhia das Letras, 2019.

KUREBAYASHI, L. F. S. et al. Fitoterapia chinesa para reduzir o estresse, a ansiedade e melhorar a qualidade de vida: estudo controlado randomizado. Rev. Esc. Enferm. USP, São Paulo, v. 50, n. 5, p. 853-860, 2016. DOI 10.1590/s0080-623420160000600020.

LOCATELLI, D. A. et al. Cooked garlic and antioxidant activity: Correlation with organosulfur compound composition. Food Chem., Barking, v. 1, n. 220, p. 219-224, 2017. DOI 10.1016/j.foodchem.2016.10.001.

LUZAK, A. et al. Association of physical activity with lung function in lung-healthy German adults: results from the KORA FF4 study. BMC Pulm. Med., Londn, v. 17, n. 1, p. 215-230, 2017. DOI 10.1186 / s12890-017-0562-8.

MACEDO, L. L.; VIMERCATI, W. C.; ARAÚJO, C. S. Fruto-oligossacarídeos: aspectos nutricionais, tecnológicos e sensoriais. Braz. J. Food Technol., Campinas, v. 23, p. e2019080, 2020. DOI 10.1590/1981-6723.08019.

MARKOWIAK, P.; SLIZEWSKA, Ż. K. Effects of probiotics, prebiotics, and synbiotics on human health. Nutrients, Basel, v. 9, n. 9, p. 1021, 2017. DOI 10.3390/nu9091021. 
MARTINS, J.; VERÍSSIMO, M. L. Ó. R. Conhecimentos e práticas de trabalhadoras de creches municipais relativos ao cuidado da criança com infecção respiratória aguda.

Interface, Botucatu, v. 10, n. 20, p. 487-504, 2006. DOI 10.1590/S008062342006000100011 .

MOLONEY, R. D. et al. The microbiome: stress, health and disease. Mamm. Genome, New York, v. 25, n. 1-2, p. 49-74, 2014. DOI 10.1007/s00335-013-9488-5.

MORENO DEL CASTILLO, M. C.; VALLADARES-GARCIA, A. J.; HALABE-CHEREM, J. Microbioma humano. Rev. Fac. Med. (Méx.), Cidade do México, v. 61, n. 6, p. 7-19, 2018. DOI 10.22201.fm.24484865e.2018.61.6.02.

MOURA, P. G.; BATISTA, L. R. V.; MOREIRA, E. A. M. População indígena: uma reflexão sobre a influência da civilização urbana no estado nutricional e na saúde bucal. Rev. Nutr., Campinas, v. 23, n. 3, p. 459-465, 2010. DOI 10.1590/S1415-52732010000300013.

PAIVA, A. C. et al. Postprandial effect of fresh and processed orange juice on the glucose metabolism, antioxidant activity and prospective food intake. J. Funct. Foods, Amsterdam, v. 52, p. 302-309, 2019. DOI 10.1016/j.jff.2018.11.013.

PEREIRA, I. F. S.; GONZAGA, M. R.; LYRA, C. O. Indicador multidimensional de fatores de risco relacionados ao estilo de vida: aplicação do método Grade of Membership. Cad. Saúde Pública, Rio de Janeiro, v. 35, n. 6, p. e00124718, 2019. DOI 10.1590/0102$311 X 00124718$.

PIMENTEL, T. C.; GARCIA, S.; PRUDENCIO, S. H. Aspectos funcionais, de saúde e tecnológicos de frutanos tipo inulina. Bol. Cent. Pesqui. Process. Aliment., Curitiba, v. 30, n. 1, p. 103-118, 2012. DOI 10.5380/cep.v30i1.28593.

RAIOL, R. A. Praticar exercícios físicos é fundamental para a saúde física e mental durante a Pandemia da COVID-19. Braz. J. Health Rev., Curitiba, v. 3, n. 2, p. 2804-2813, 2020. DOI 10.34119/bjhrv3n2-124.

SAMPAIO, M. Reabilitação respiratória: a abordagem holística da pessoa com DPOC. Rev. Port. Med. Geral Fam., Lisboa, v. 35, n. 2, p. 151-157, 2019. DOI 10.32385/rpmgf.v35i2.12013.

SANCHEZ, M.; PANAHI, S.; TREMBLAY, A. Childhood obesity: a role for gut microbiota? Int. J. Environ. Res. Public Health, Basel, v. 12, n. 1, p. 162-175, 2015. DOI 10.3390/ijerph120100162.

SANTOS, F. L. Os alimentos funcionais na mídia: quem paga a conta. In: PORTO, C. M.; BROTAS, A. M. P.; BORTOLIERO, S. T. Diálogos entre ciência e divulgação científica: leituras contemporâneas. Salvador: EDUFBA, 2011. p. 199-210.

SARAIVA, G. L. et al. Prevalência da deficiência, insuficiência de vitamina D e hiperparatiroidismo secundário em idosos institucionalizados e moradores na comunidade da cidade de São Paulo, Brasil. Arq. Bras. Endocrinol. Metab., São Paulo, v. 51, n. 3, p. 437442, 2007. DOI 10.1590/S0004-27302007000300012.

SCHIVINSKI, C. et al. Brinquedos terapêuticos: a sobreposição dos estímulos ventilatórios altera a mecânica respiratória de crianças saudáveis? Rev. Paul. Pediatr., São Paulo, v. 38, p. e2018259, 2020. DOI 10.1590/1984-0462/2020/38/2018259. 
SHORI, A. B. Influence of food matrix on the viability of probiotic bacteria: A review based on dairy and non-dairy beverages. Food Biosci., Amsterdam, v. 13, p. 1-8, 2016.

SILVA, A. C. C. et al. Alimentos contendo ingredientes funcionais em sua formulação:

Revisão de artigos publicados em revistas brasileiras. Conex. Ciênc., Formiga, v. 11, n. 2, p. 134-144, 2016.

SKARPSNO, E. S. et al. The joint effect of insomnia symptoms and lifestyle factors on risk of self-reported fibromyalgia in women: longitudinal data from the HUNT Study. BMJ

Open, London, v. 9, n. 8, p. e028684, 2019. DOI 10.1136/bmjopen-2018-028684.

SKARPSNO, E. S. et al. Work-related mental fatigue, physical activity and risk of insomnia symptoms: longitudinal data from the Norwegian HUNT study. Behav. Sleep Med., London, v. 15, p. 1-12, 2020. DOI 10.1080/15402002.2019.1614927.

STIES, S.W. et al. Influence of exercise on oxidative stress in patients with heart failure.

Heart Fail Rev., Norwell, v. 23, p. 225-235, 2018. DOI 10.1007/s10741-018-9686-z.

STORCK, C. R. et al. Folhas, talos, cascas e sementes de vegetais: composição nutricional, aproveitamento na alimentação e análise sensorial de preparações. Cienc. Rural, Santa Maria, v. 43, n. 3, p. 537-543, 2013. DOI 10.1590/S0103-84782013000300027.

VALENTINI, J. N. et al. Efeitos da suplementação simbiótica no funcionamento do intestino e na inflamação sistêmica de idosos residentes em comunidades - análises secundárias de um teste clínico aleatório. Arq. Gastroenterol., São Paulo, v. 57, n. 1, p. 24-30, 2020. DOI 10.1590/s0004-2803.202000000-06.

VALENZUELA, P. L. et al. Lifelong endurance exercise as a countermeasure against agerelated [Formula: see text] decline: physiological overview and insights from masters athletes Sports Med., Auckland, v. 50, n. 4, p. 703-716, 2020. DOI 10.1007 / s40279-019-01252-0.

VIDAL, M. A. et al. A ingestão de alimentos funcionais e sua contribuição para a diminuição da incidência de doenças. Cad. Grad. Ciênc. Biol. Saúde, Aracaju, v. 1, n. 15, p. 43-52, 2012.

WORLD HEALTH ORGANIZATION. Food and Agriculture Organization of the United Nations. COVID-19 and food safety: guidance for food businesses. Geneva: WHO \& FAO, 2020 .

YWASSAKI, L. A.; CANNIATTI-BRAZACA, S. G. Ácido ascórbico e pectina em diferentes tamanhos e partes de frutas cítricas. Ciênc. Tecnol. Aliment, Campinas, v. 31, n. 2, p. 319$326,2011$.

ZHAO, F. et al. Pomegranate extract and exercise provide additive benefits on improvement of immune function by inhibiting inflammation and oxidative stress in high-fat-diet-induced obesity in rats. J. Nutr. Biochem., New York, v. 32, p. 20-28, 2016. DOI 10.1016/j.jnutbio.2016.02.003. 


\title{
KNOWLEDGE DIALOGUES IN HEALTH PROMOTION AND PREVENTION OF COVID-19 RELATED DISEASES
}

\begin{abstract}
This study attempted to reflect on health promotion and prevention using diferente types of knowledge, in the context of the COVID-19 pandemic. We carried a bibliography review based on scientific evidence and traditional knowledge from a multisystemic health perspective. It included nutritional aspects, physical activity, and integrality of health care in a holistic approach, transcending scientifically produced understanding for the discussion of traditional knowledge and other cultural elements of extreme importance. Because of the accumulated information in health and nutrition, a reflection of the biocultural arsenal is emphasized in the comprehension of integral health in confronting the demands resulting from the pandemic.
\end{abstract}

Keywords: pandemic; health promotion; nutrition; food culture; knowledge integration.

O presente trabalho foi realizado com apoio da Coordenação de Aperfeiçoamento de Pessoal de Nível Superior - Brasil (CAPES) - Código de Financiamento 001. 